\title{
Descargas Atmosféricas e Condições Ambientais Associadas a Convecção Profunda: um Caso de Estudo
}

\author{
Lightning and Environmental Conditions Associated with Deep Convection: A Case \\ Study
}

\author{
Luciana Cardoso Neta*1 $^{*}$ Gabriela Lameu Moreira ${ }^{2}$ e Roseli Gueths Gomes ${ }^{3}$ \\ ${ }^{1}$ Doutorando em meteorologia, Universidade Federal de Santa Maria , RS, Brasil \\ ${ }^{2}$ Mestranda, Universidade Federal de Pelotas, RS, Brasil \\ ${ }^{3}$ Professora, Doutora em Física da Atmosfera, Universidade Federal de Pelotas, RS, Brasil
}

\begin{abstract}
Resumo
Neste trabalho investiga-se a incidência de descargas elétricas atmosféricas, precipitação em superfície e condições ambientais em associação a um sistema linear observado em Minas Gerais, entre os dias 17-18 de outubro de 2007. Para isto, foram utilizadas imagens de satélite geoestacionário, dados da RINDAT e de estações automáticas do INMET. As condições ambientais foram obtidas com o modelo WRF. As descargas ocorreram de maneira alinhada às áreas de convecção mais intensa deste sistema e em maior quantidade na parte norte. A quantidade total de precipitação em superfície foi de 135,2mm durante o tempo de vida de, aproximadamente, 20 horas deste sistema. Observou-se que a região onde o sistema linear se formou e se desenvolveu estava propícia para a ocorrência de tempestades, apresentando grande umidade, confluência de ventos em determinados horários, o que favoreceu ainda mais a convecção.
\end{abstract}

Palavras-chave: Sistema convectivo. Atividade elétrica. Índices de instabilidade

\begin{abstract}
In this work we investigate the impact of lightning, surface precipitation and environmental conditions in combination with a linear system observed in Minas Gerais, between 17-18 October 2007. It was used geostationary satellite imagery, lightning data from RINDAT network and data from automatic stations INMET. The environmental conditions were obtained from the WRF model. Lightning were aligned to the areas of most intense convection of this system and in greater quantity in the northern part. The total quantity of surface precipitation was $135.2 \mathrm{~mm}$ during the lifetime of approximately 20 hours of this system. It was observed that the region where the linear system formed and evolved was favorable for the occurrence of storms, with high humidity and confluence of winds at certain times, which favored further convection.
\end{abstract}

Keywords: Convective system. Electrical activity. Instability index 


\section{Introdução}

$\mathrm{O}$ Sudeste Brasileiro, por causa de sua posição geográfica, tem as condições de tempo influenciadas por sistemas meteorológicos tanto de latitudes médias quanto baixas (MINUZZI et al., 2007). No verão austral, o escoamento de altos níveis sobre a América do Sul é marcado pela presença da Alta da Bolívia (LENTERS \& COOK, 1999) e dos Vórtices Ciclônicos de Altos Níveis tropicais e, em baixos níveis, pelos sistemas de baixa pressão no Noroeste da Argentina e na região do Chaco (ESCOBAR \& SELUCHI, 2012).

Os totais pluviométricos na região Sudeste são máximos no verão e mínimos no inverno, o que caracteriza o regime de monção na América do Sul (ZHOU \& LAU, 1998). Parte da umidade que atinge o Sudeste brasileiro é proveniente da Amazônia, pelo Jato de Baixos Níveis, localizado a leste da Cordilheira dos Andes (MARENGO et al., 2004). Quando este escoamento converge com aquele proveniente da Alta Subtropical no Oceano Atlântico Sul, uma banda de nebulosidade quase estacionária se forma sobre as regiões Centro-Oeste e Sudeste do Brasil, orientada no sentido NW-SE, caracterizando a Zona de Convergência do Atlântico Sul (QUADRO, 1994), que tem um papel fundamental na intensificação da precipitação sobre a região Sudeste do Brasil (KODAMA, 1992). Além destes eventos meteorológicos, são frequentes as formações de sistemas convectivos de mesoescala (SCM), associados ao aquecimento continental (MINUZZI et al., 2007). Estes sistemas podem provocar condições adversas de tempo em superfície, tais como rajadas de vento, queda de granizo e descargas elétricas atmosféricas.

$\mathrm{O}$ estado de Minas Gerais (MG) é o segundo mais populoso do Brasil e está situado em um planalto, onde as altitudes variam entre 100 e $1500 \mathrm{~m}$. Nas Serras da Mantiqueira, do Espinhaço, da Canastra e do Caparaó estão localizadas as maiores altitudes, nas quais há terrenos localizados acima de $1700 \mathrm{~m}$. As serras apresentam declividades consideráveis e há forte influência orográfica nas diferentes condições climáticas.

Reis (2005) realizou um estudo climatológico das descargas atmosféricas detectadas em MG e destacou a ocorrência do que chamou de "cinturão" de incidência de descargas atmosféricas, entre as Serras da Canastra e da Mantiqueira, onde a formação de tempestades é frequente. As descargas elétricas atmosféricas ocorrem devido ao acúmulo de cargas elétricas em regiões localizadas da atmosfera, geralmente no interior das tempestades. As descargas tem início quando o campo elétrico produzido por estas cargas excede a capacidade isolante do ar (também conhecida como rigidez dielétrica) em um dado local na atmosfera, que pode ser dentro da nuvem ou próximo ao solo (PINTO JR., 2005).

O número de descargas detectadas por hora pode revelar importantes características da evolução de sistemas convectivos e ocorrer tanto nas áreas convectivas quanto nas regiões estratiformes (LANG \& RUTLEDGE,
2002). Uma vez que dados de descargas fornecem uma indicação direta da atividade convectiva em nuvens, podem ser utilizados no monitoramento de condições que conduzam à ocorrência de tempo severo em superfície, como mostrado por Schultz et al. (2011). O uso de dados de descargas nuvem-solo se mostrou eficiente para aplicações meteorológicas específicas e sociais (por exemplo, segurança humana e proteção de infraestrutura).

A integração da observação de sistemas convectivos via sensoriamento remoto (por exemplo, com imagens de satélite) com dados de descargas pode constituir uma ferramenta muito útil em trabalhos com propósitos tanto diagnósticos quanto prognósticos da intensidade da atividade convectiva (FORMENTON et al., 2013). Dada a grande variabilidade de tipos de sistemas convectivos e de diferentes padrões de descargas atmosféricas, estudos de casos permitem discernir características individuais em sistemas convectivos.

Para uma correta avaliação do desenvolvimento e da evolução de tais sistemas é importante considerar tanto os aspectos termodinâmicos quanto os aspectos dinâmicos das tempestades. Em relação aos aspectos termodinâmicos devem ser avaliadas as condições de estabilidade/instabilidade de acordo com o tipo de atmosfera que está sendo considerada. Quanto mais quente e úmido for o ar ambiente em níveis baixos e relativamente mais seco em níveis médios, a situação ambiental será cada vez mais favorável aos mecanismos de desenvolvimento de células convectivas.

Dentro deste contexto, pretende-se analisar as distribuições espaciais e temporais das descargas atmosféricas, precipitação em superfície e condições atmosféricas de mesoescala associadas à ocorrência de um sistema linear no estado de Minas Gerais, entre os dias 17 e 18 de outubro de 2007. Este sistema se formou entre as Serras de Canastra e de Mantiqueira, bem na região denominada como "cinturão" de descargas atmosféricas (REIS, 2005).

\section{Materiais e Métodos}

Foram utilizadas imagens de satélite geoestacionário da série GOES, obtidas junto ao Centro de Previsão do Tempo e Estudos Climáticos (CPTEC/INPE), para identificar o sistema linear em estudo. Os dados de descargas atmosféricas foram detectados pelos sensores da RINDAT (Rede integrada Nacional de Detecção de Descargas), que detectam descargas que partem da nuvem e se deslocam em direção ao solo (descargas negativas) ou o contrário (descargas positivas). O sistema linear ocorreu na região onde a eficiência de detecção desta rede é igual ou superior a 90\% (NACCARATO \& PINTO JR., 2008). O total de descargas atmosféricas detectadas em intervalos de 30 e 10 minutos foi sobreposto nas imagens de satélite disponíveis desde o momento de 
formação até a dissipação do sistema. A contabilização das descargas provocadas pelo sistema em estudo foi efetuada pela identificação do mesmo por uma área retangular, dentro da qual as descargas foram totalizadas separadamente do restante da região (CARDOSO NETA \& GOMES, 2013).

Para a confecção dos mapas de precipitação, foram usados os dados horários obtidos das estações meteorológicas automáticas de superfície do INMET (Instituto Nacional de Meteorologia) localizadas na região de ocorrência do sistema linear em estudo.

Os campos de razão de mistura (a $2 \mathrm{~m}$ ) e do vento horizontal (em $850 \mathrm{hPa}$ ) e dos índices convectivos ( $\mathrm{K}$ e SWEAT) foram obtidos a partir dos dados oriundos das simulações com o modelo WRF (SKAMAROCK et al., 2008), utilizando 3 grades aninhadas, como mostra a Fig.1. O domínio maior, com $36 \mathrm{~km}$ de resolução horizontal, envolve o Brasil e se estende até o sul da América do Sul, incluindo também o Oceano Atlântico até $30^{\circ} \mathrm{W}$. O domínio intermediário, com $12 \mathrm{~km}$ de resolução horizontal, abrange vários estados localizados nas regiões Sudeste, Centro-Oeste e Sul do Brasil, principalmente. O domínio menor, com $4 \mathrm{~km}$ de resolução horizontal, limita-se aos estados localizados na região Sudeste do Brasil. Foram utilizadas as mesmas opções das parametrizações físicas nas três grades, baseadas nos resultados obtidos por Zepka (2011). As simulações tiveram início às 12:00UTC do dia 16, com 35 níveis verticais e dados de entrada FNL (Final Analysis) atualizados a cada 6 horas, com $1^{\circ}$ de resolução em latitude e longitude.

Definição dos índices convectivos calculados:

\section{a) Índice $K$}

$\mathrm{O}$ índice $\mathrm{K}\left({ }^{\circ} \mathrm{C}\right)$ representa uma medida potencial de desenvolvimento de tempestades, baseada na taxa vertical de variação de temperatura, no conteúdo de umidade na baixa troposfera e na extensão vertical da camada úmida. Segundo George (1960), o índice K é definido como:

$$
K=T_{850}+T d_{850}-T_{500}-\operatorname{Dep}_{700}
$$

onde T, Td e Dep são respectivamente temperatura, temperatura do ponto de orvalho e depressão do bulbo úmido ambientais (em graus Celsius) para os níveis de pressão indicados em subscrito. Assim, o índice K leva em conta a taxa de variação vertical de temperatura (TVVT) de níveis médios, a umidade em $850 \mathrm{hPa}$, e o nível de secura atmosférica em 700hPa (NASCIMENTO, 2005). Para ser significativo deve haver calor em baixos níveis e umidade em abundância desde a superfície até $700 \mathrm{hPa}$. Uma camada seca em $700 \mathrm{hPa}$ reduz o valor de $\mathrm{K}$. Valores de $\mathrm{K}$ acima de $30^{\circ} \mathrm{C}$ são considerados altos e com alto potencial para ocorrência de tempestades, e acima de $40^{\circ} \mathrm{C}$ são extremamente altos com potencial extremo para tempestades (HENRY, 1987).

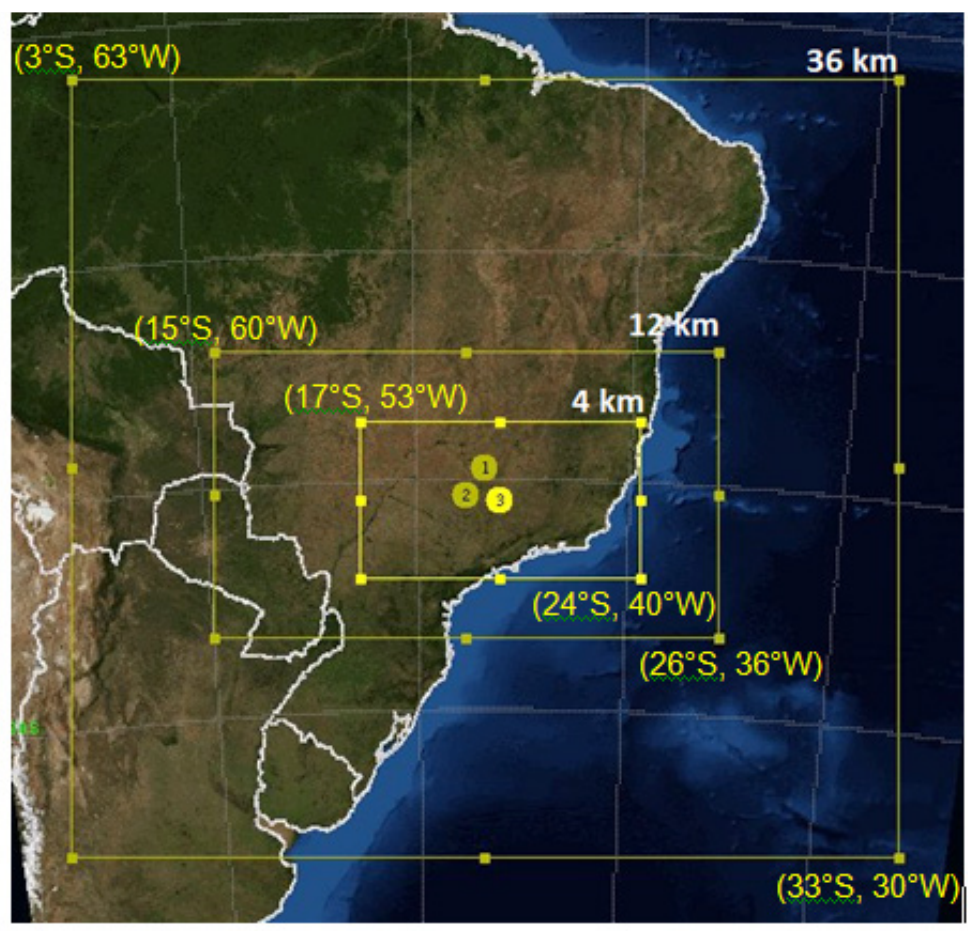

Figura 1 - Localização dos três domínios de processamento com o modelo WRF, com as resoluções horizontais e limites geográficos respectivos. 


\section{b) Índice Severe Weather Threat (SWEAT)}

O índice SWEAT combina informações sobre as estruturas termodinâmica e cinemática da atmosfera sendo, portanto, desenvolvido para a previsão específica de potencial para tempestades severas. A equação do índice SWEAT, adaptada para o hemisfério sul, é dada por (NASCIMENTO, 2005):

$$
\begin{array}{r}
\text { SWEAT }=20(T T-49)+12 T d_{850}+2 V_{850}+ \\
V_{500}+125(\text { sen } \mid \text { diffdir } \mid+0.2)
\end{array}
$$

onde TT $\left({ }^{\circ} \mathrm{C}\right)$ é o índice Total-Tottals definido como (MILLER, 1972):

$$
T T=T_{850}+2 T d_{850}-2 T_{500}
$$

os termos $\mathrm{V}_{850}$ e $\mathrm{V}_{500}$ são a velocidade do vento (em nós) para os níveis de $850 \mathrm{hPa}$ e $500 \mathrm{hPa}$, respectivamente. O termo diffdir representa a diferença entre as direções do vento (em graus) entre $500 \mathrm{hPa}$ e $850 \mathrm{hPa}$, ou seja, diffdir $=$ direçãoV ${ }_{500}-$ direção $V_{850}$. Se o valor de TT for menor que 49 e $\mathrm{Td}_{850}$ for menor que zero estes termos deverão ser igualados a zero. Outras considerações a respeito do índice SWEAT podem ser encontradas em Nascimento (2005).

\section{Resultados}

O sistema linear em análise ocorreu entre 17:00UTC do dia 17 até 14:00UTC do dia 18 de outubro de 2007, aproximadamente, totalizando 21 horas de tempo de vida. A evolução deste sistema é mostrada na Fig.2, onde foram sobrepostas as descargas detectadas em 30 minutos. O processo de formação deste sistema foi semelhante ao denominado como "broken line", por Bluestein e Jain (1985), pois a disposição dos elementos convectivos estava fragmentada inicialmente (Fig.2a e $2 b$ ) para se unir formando uma linha contínua. Percebe-se que durante todo o tempo de vida deste sistema linear, não foram observadas outras formações convectivas intensas na sua vizinhança. Três horas mais tarde (Fig.2c) a quantidade de descargas mais do que triplicou e se posicionaram ao longo dos núcleos convectivos mais intensos. Cerca de $90 \%$ das descargas detectadas foram geradas nas primeiras 9 horas do ciclo de vida deste sistema e se concentraram nas áreas com temperatura do topo das nuvens $\leq-50^{\circ} \mathrm{C}$. Durante o ciclo de vida deste sistema linear, que gerou quase 7.000 descargas, o valor máximo de descargas ocorreu entre 0:30UTC e 1:30UTC do dia 18(Fig.2d), com 2.341 descargas. Às 4:30 (Fig.2e) este sistema linear iniciou sua dissipação a partir do setor leste. Entretanto, ainda foi possível observar um núcleo no setor oeste com topo frio, a $-60^{\circ} \mathrm{C}$. A Fig. $2 \mathrm{f}$
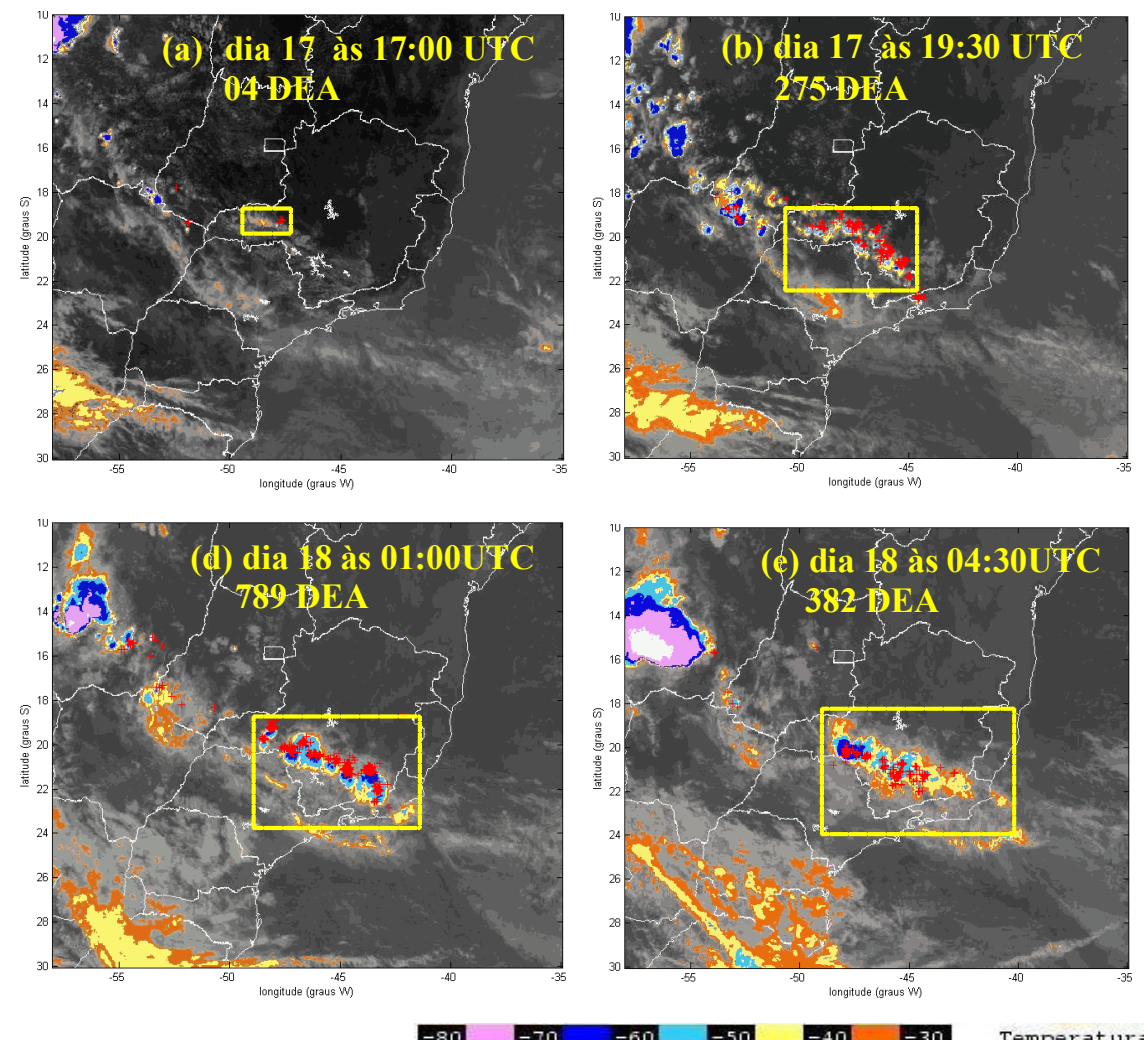
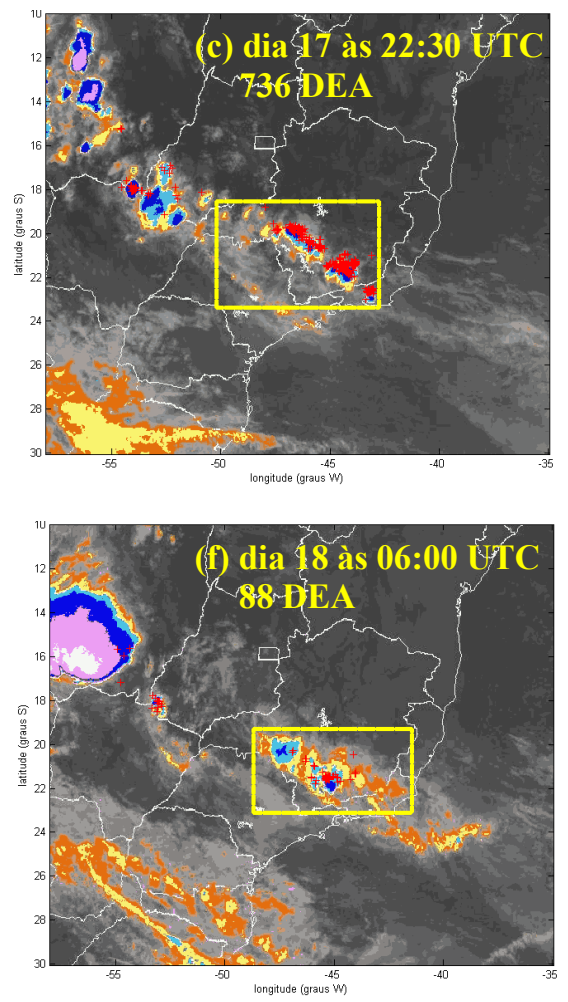

Celsius

Figura 2 - Segmentos das imagens de satélite, com o total de descargas elétricas atmosféricas (DEA, cruzes em vermelho) detectadas em 30 minutos. 


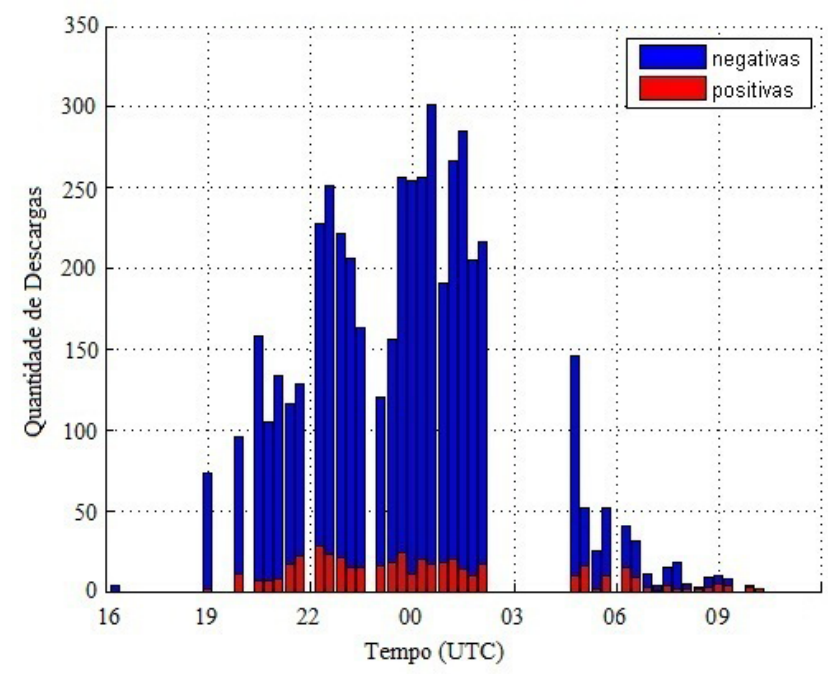

Figura 3 - Distribuição temporal das descargas atmosféricas negativas (azul) e positivas (vermelho)

(das 06:00UTC) mostra a dissipação do sistema linear, pois observam-se nuvens com topo baixo, indicando seu enfraquecimento.

A distribuição temporal das descargas atmosféricas associadas a este sistema linear, considerando as 50 imagens de satélite disponíveis durante o período completo de sua avaliação e com a sobreposição das descargas considerando 10 minutos, é mostrada na Fig. 3. As lacunas existentes mostram as falhas nas sequências das imagens de satélite. De maneira geral, observa-se no histograma a predominância de descargas negativas durante todo o período de acompanhamento do sistema, com exceção de alguns períodos na fase da dissipação. Segundo Watson et al. (1994) e Macgorman \& Burges (1994), as descargas negativas superam as positivas quando as precipitações são predominantemente de água líquida e, quando existe muita precipitação na forma de granizo, as descargas positivas tendem a ocorrer em maior quantidade. Nas cinco horas finais do sistema foi detectada uma descarga por imagem de satélite.

A quantidade de precipitação em superfície causada pelo sistema linear em estudo foi analisada com os dados de 21 estações automáticas do INMET. Estas estações foram selecionadas por estarem localizadas onde o sistema linear foi observado. A Fig.4 mostra os totais horários de precipitação (barras) e de descargas atmosféricas (linha), desde o início até o término do sistema linear. Observa-se que a quantidade máxima de descargas ocorreu uma hora depois do máximo de precipitação. Na segunda metade do ciclo de vida deste sistema linear, a quantidade de descargas detectadas foi mínima, quando também diminuiu a precipitação em superfície, com exceção do horário entre 06:00 e 07:00UTC, quando $26,3 \mathrm{~mm}$ de precipitação foram registrados. Este valor ocorreu no sul de Minas Gerais, provocado pela intensificação de um dos núcleos convectivos do sistema linear (ver Fig. 2f)

A evolução temporal e espacial das descargas atmosféricas detectadas pelo sistema linear em estudo é mostrada na Fig.5. Na Fig.5a são mostradas as descargas detectadas em cada um dos 4 períodos em que foi dividido o ciclo de vida do sistema linear. As descargas detectadas no primeiro quarto de vida estão plotadas em amarelo, as que ocorreram nas 5 horas seguintes o

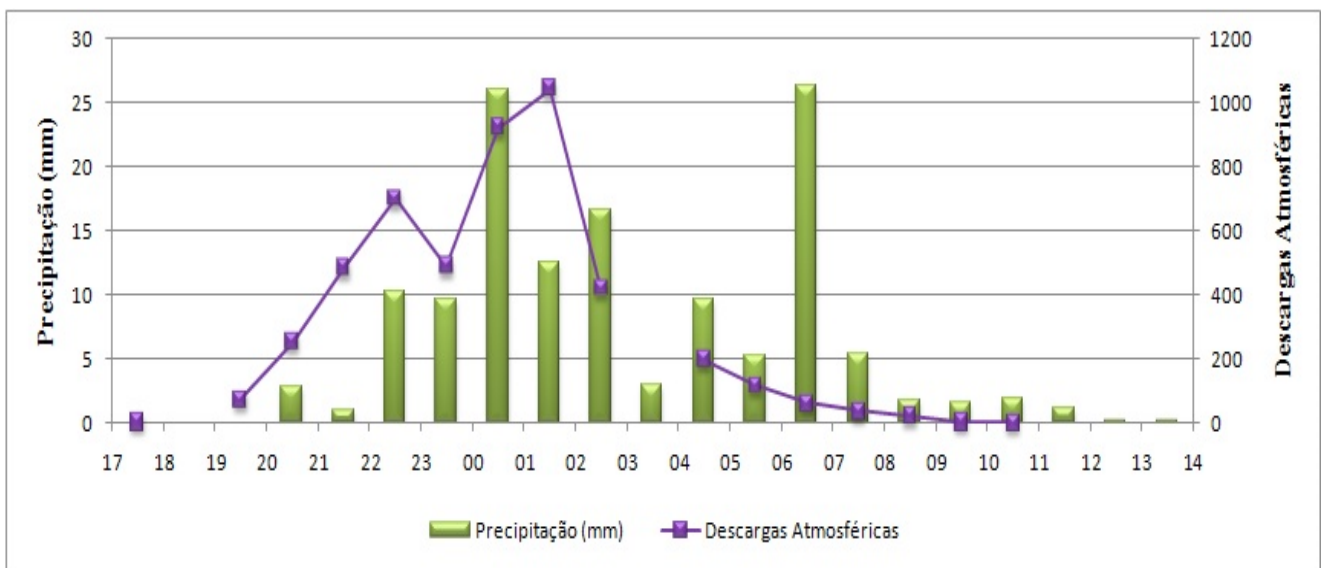

Figura 4 - Distribuição horária das descargas atmosféricas (linha) e da precipitação em superfície (barras). 

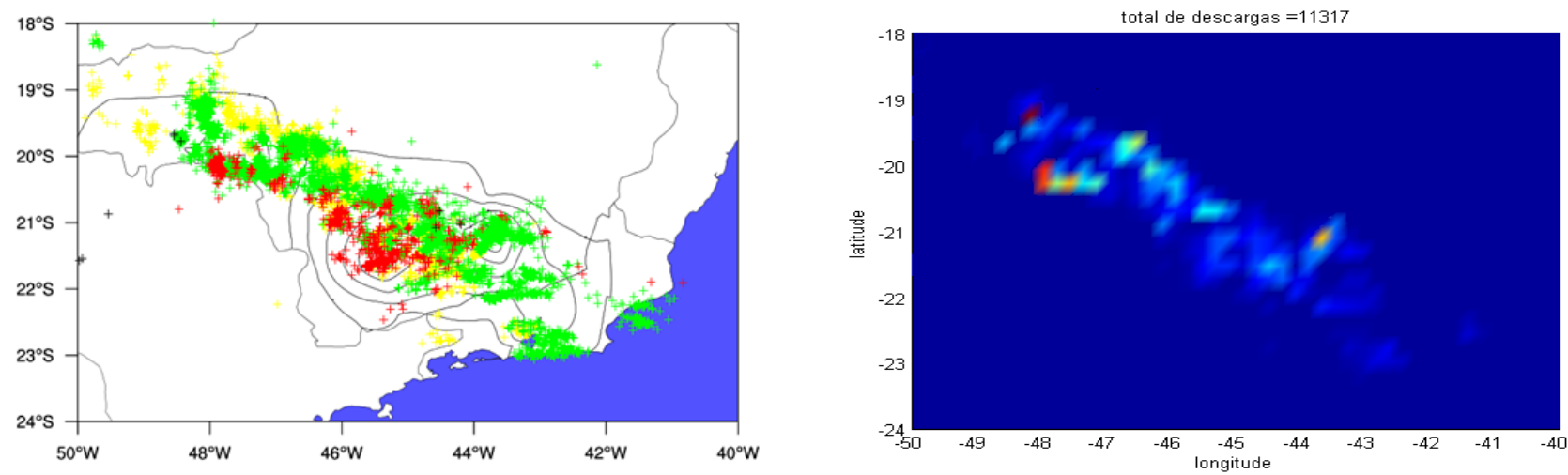

Figura 5 - (a) Total de descargas atmosféricas plotadas em 4 intervalos de 5 horas e precipitação total (isolinhas), (b) densidade do total de descargas (descargas $/ 400 \mathrm{~km}^{2}$ ).

estão em verde, seguidas das descargas em vermelho e, finalmente, as descargas detectadas no último quarto de vida do sistema linear, estão plotadas na cor preta. Ainda, estão indicadas as isolinhas de precipitação total em superfície. A Fig.5b mostra o campo de densidade de descargas (descargas $/ 400 \mathrm{~km}^{2}$ ) contabilizado sobre todo o tempo de vida do sistema linear. Nota-se o alinhamento das descargas atmosféricas com a nebulosidade. Na região onde a precipitação em superfície foi mais abundante, ocorreram descargas até o término do sistema linear.
Entretanto, a maior quantidade de descargas ocorreu na extremidade norte do sistema linear como mostra a Fig.5b.

As condições ambientais são mostradas na Fig.6, desde 19:00UTC do dia 17 até 04:00UTC do dia 18, em intervalos de 3 horas. Observa-se (Fig.6a) a razão de mistura variando entre valores menores que $8 \mathrm{~g} / \mathrm{kg}$ até $12 \mathrm{~g} / \mathrm{kg}$ na região onde o sistema linear estava se formando. Com o desenvolvimento do sistema linear (Fig.6b e 6c), a razão de mistura continuou apresentando valores inferiores a

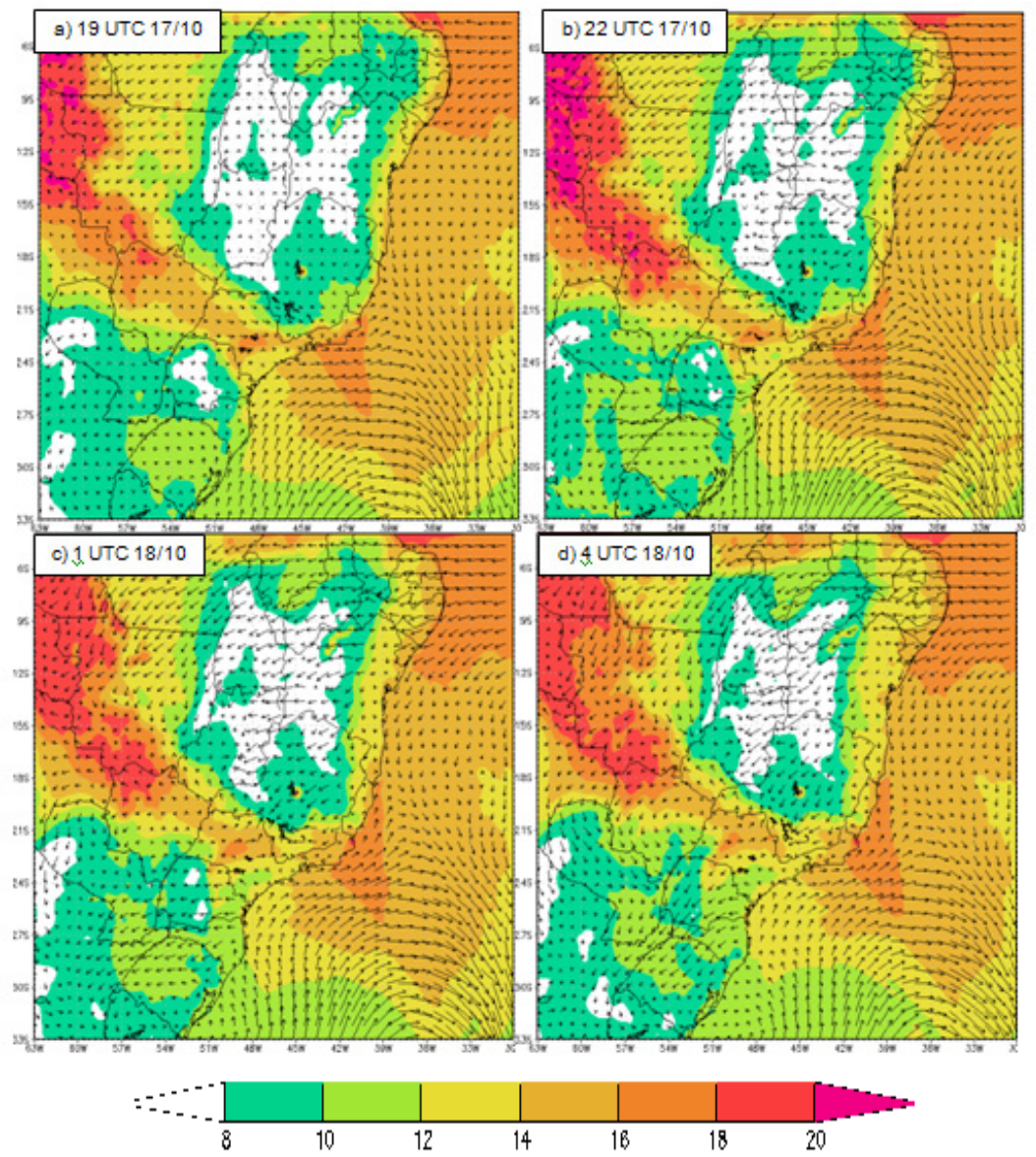

Figura 6 - Campos de razão de mistura a $2 \mathrm{~m}$ de altura (hachurado) e vetor vento horizontal no nível $850 \mathrm{hPa}$, obtidos com o domínio maior (36km de resolução horizontal). 


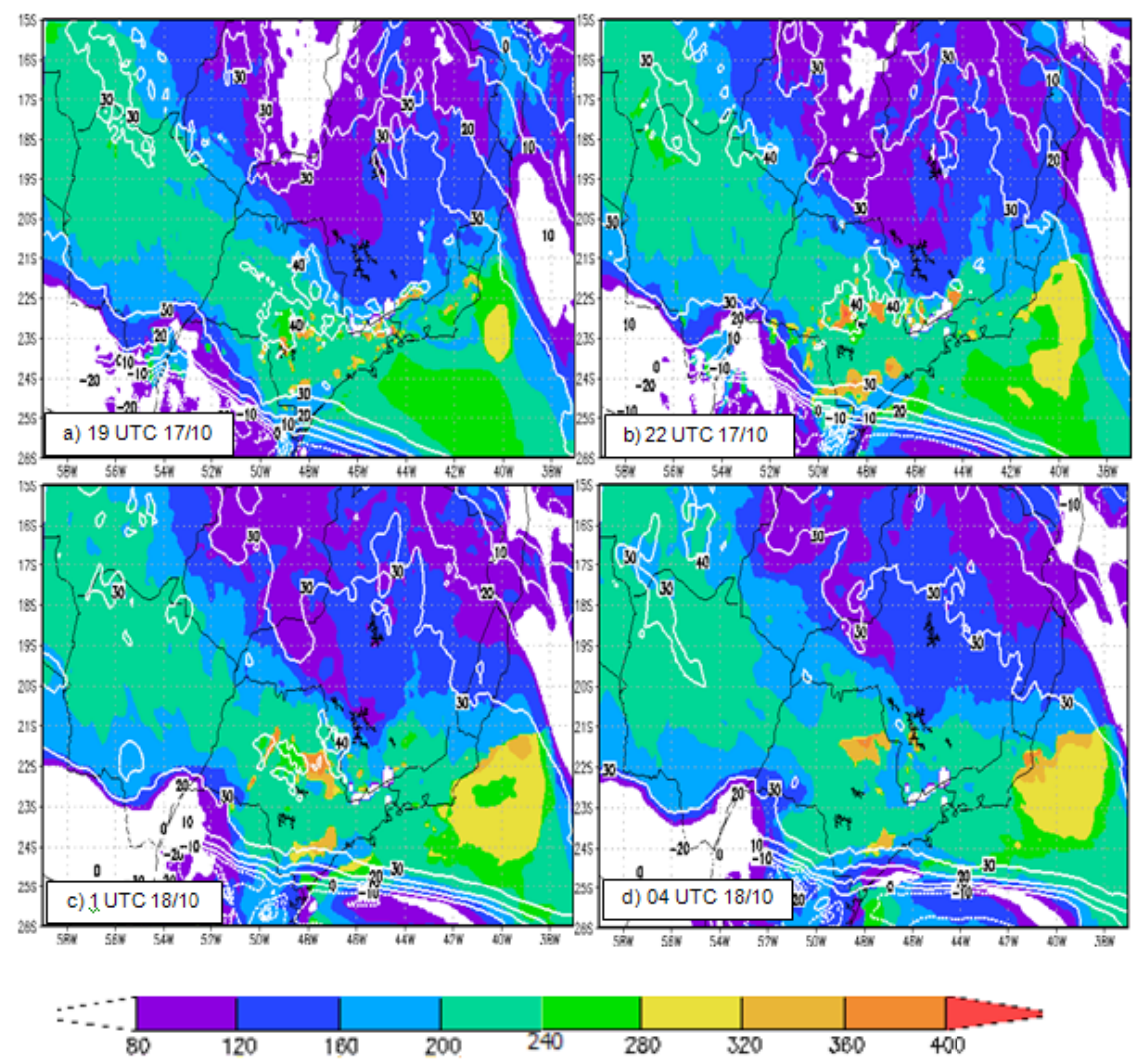

Figura 7 - Campos dos índices K (isolinhas) e SWEAT (hachurado), obtidos com o domínio de 12km de resolução horizontal.

$8 \mathrm{~g} / \mathrm{kg}$ até $14 \mathrm{~g} / \mathrm{kg}$, porém as áreas com valores maiores começaram a aumentar pelo estado de SP e sul de MG.

Os campos de vento nestes horários eram similares, apresentando uma pequena área de confluência ao sul de MG, onde o sistema linear estava atuando. Esta confluência pode ter auxiliado na convecção e desenvolvimento do sistema. Às 04:00UTC (Fig.6d), a região sul de MG ficou um pouco mais úmida, com os valores de razão de mistura entre $8 \mathrm{~g} / \mathrm{kg}$ a $14 \mathrm{~g} / \mathrm{kg}$, porém em áreas um pouco maiores sobre a região. O vento ainda apresentava uma configuração de confluência. Posteriormente, o sistema linear já estava bastante enfraquecido, mas a região ainda apresentava os mesmos valores de razão de mistura e o vento não mais apresentava confluência.

Nas Fig.7a e 7b pode-se observar que os valores de SWEAT estavam entre 80 e 200 na região onde o sistema linear se formou e desenvolveu. Porém, notam-se algumas áreas próximas com valores que chegaram a aproximadamente 360, indicando que esta região estava instável e propícia à ocorrência de tempestades fortes. $\mathrm{O}$ índice $\mathrm{K}$ se manteve variando de 30 a $40^{\circ} \mathrm{C}$ neste período. $\mathrm{Na}$ Fig.7c à 01:00UTC do dia 18, a configuração se manteve. $\mathrm{Na}$ Fig.7d os valores de ambos os índices diminuíram, o índice $\mathrm{K}$ ficou em $30^{\circ} \mathrm{C}$ e o índice SWEAT retornou aos valores iniciais, variando de 80 a 200, indicando que, conforme o sistema enfraquecia e entrava em dissipação, a instabilidade do ambiente diminuía.

\section{Conclusões}

Neste trabalho foram apresentadas as variações temporais e espaciais das descargas elétricas atmosféricas provocadas por um sistema linear ocorrido em Minas Gerais, durante o período compreendido entre os dias 17 e 18 de outubro de 2007. Este intervalo de tempo foi dividido em períodos de 3 horas, nos quais foram avaliadas as quantidades de descargas atmosféricas e de precipitação geradas em superfície e alguns campos meteorológicos obtidos com o modelo WRF. As variáveis de grande escala foram analisadas com o domínio de $36 \mathrm{~km}$ de resolução horizontal, e as de mesoescala o foram com o domínio de $12 \mathrm{~km}$.

As descargas atmosféricas se posicionaram de maneira alinhada com os núcleos convectivos imersos na nebulosidade deste sistema. A geração das descargas acompanhou o ciclo evolutivo. A região onde o sistema linear se formou e se desenvolveu estava propícia para a ocorrência de tempestades, apresentando grande umidade, confluência de vento em determinados horários que favorecia ainda mais a convecção. Os índices de instabilidades com valores altos, algumas vezes indicativos a evento severo, foram compatíveis com a atividade convectiva observada. 


\section{Agradecimentos}

Os autores agradecem à Empresa Furnas pelo fornecimento dos dados de descargas elétricas atmosféricas.

\section{Referências}

BLUESTEIN, H. B.; JAIN, M. Formation of mesoscale lines of precipitation: severe squall lines in Oklahoma during the spring. Journal of the Atmospheric Sciences, v. 42, p. 1711-32, 1985.

CARDOSO NETA, L. ; GOMES, R.G. Precipitation on the surface and cloudto-ground lightning during a storm in southeastern Brazil. In: INTERNATIONAL SYMPOSIUM ON LIGHTNING PROTECTION, 12, 2013, Belo Horizonte, MG. Anais do... Belo Horizonte, 2013.

ESCOBAR, G. C.; SELUCHI, M.E. Classificação sinótica dos campos de pressão atmosférica na América do Sul e sua relação com as baixas do chaco e do noroeste argentino. Revista Brasileira de Meteorologia, v. 27, n.3, p.365-375, 2012.

FORMENTON, M.; PANEGROSSI, G.; CASELLA, D.; DIETRICH, S.; MUGNAI, A.; SANO, P.; PAOLA, F.D.; BETZ, H-D.; PRICE, C.; YAIR, Y. Using a cloud electrification model to study relationships between lightning activity and cloud microphysical structure. Natural Hazards and Earth System Sciences, v. 13, p. 1985-1104, 2013.

GEORGE, J. J. Weather Forecasting for Aeronautics. Academic Press, 1960, 673 p.

HENRY, W. The Skew-T, Log P Diagram. National Weather Service Training Center, EUA, 1987, 68 p.

KODAMA, Y. M. Large-scale common features of subtropical precipitation zones (the Baiu Frontal Zone, the SPCZ, and the SACZ). Part I: characteristics of subtropical frontal zones. Journal of the Meteorological Society of Japan, v. 70, n. 4, p. 813-835, 1992.

LANG, T. J.; RUTLEDGE, S. A. Relationships between Convective Storm Kinematics, Precipitation, and Lightning. Monthly Weather Review, v.130, n.10, p. 2492-2506, 2002.

LENTERS, J.D.; COOK, K.H. Summertime precipitation variability over South America: role of the large-scale circulation. Monthly Weather Review, v.127, p. 409431, 1999.
MACGORMAN, D. R.; BURGESS, D. W. Positive Cloud-to-Ground Lightning in Tornadic Storm and Hailstorms. Monthly Weather Review, v.122, p.16711697, 1994.

MARENGO, J. A.; SOARES, W. R.; SAULO, A. C.; NICOLINI, M. Climatology of the Low-Level Jet East of the Andes as Devired from NCEP-NCAR Reanalysis: characteristics and temporal variability. Journal of Climate, v.17, n.12, p.2261-2280, 2004.

MILLER, R. C. Notes on analysis and severe storm forecasting procedures of the Air Force Global Weather Central. Techical Report 200, Air Weather Service, United States Air Force, 190 pp., 1972.

MINUZZI, R. B.; SEDIYAMA, G. C.; BARBOSA, E. M.; MELO Jr., J. C. F. Climatologia do comportamento do período chuvoso da região sudeste do Brasil. Revista Brasileira de Meteorologia, v.22, n.3, p.338-344, 2007.

NACCARATO, K. P.; PINTO Jr., O. The third generation relative detection efficienty model for the Brazilian Lightning Detection Network (BRASILDat). INTERNATIONAL LIGHTNING DETECTION CONFERENCE, 20, INTERNATIONAL LIGHTNING METEOROLOGY CONFERENCE, 2, Tucson, Arizona,USA, Anais do... Tucson, Arizona,USA, 2008.

NASCIMENTO, E. L. Previsão de tempestades severas utilizando-se parâmetros convectivos e modelos de mesoescala: uma estratégia operacional adotável no Brasil? Revista Brasileira de Meteorologia, v.20, n.1, p. 121-140, 2005.

PINTO JR, Osmar. A Arte da Guerra Contra os Raios. Oficina de Textos, 2005, 79p.

QUADRO, Mário Francoso Leal de. Estudo dos Episódios de Zona de Convergência do Atlântico Sul (ZCAS) sobre a América do Sul. 1994. 123f. Dissertação (Mestrado em Meteorologia) - Instituto Nacional de Pesquisas Espaciais, São José dos Campos.

REIS, Ruibran Januário dos Reis. Mapeamento a climatologia das descargas atmosféricas em Minas Gerais utilizando dados de 1989 a 2002 - uma análise exploratória. 2005. 131f. Tese (Doutorado em Geografia) -Pontifícia Universidade Católica de Minas Gerais.

SCHULTZ, C. J.; PETERSEN, W. A.; CAREY, L. D. Lightning and severe weather: A comparison between total and cloud-to-ground lightning trends, Weather and Forecasting, v.26, p. 744-755, 2011. 
SKAMAROCK, W. C.; KLEMP, J.B.; DUDHIA, J.; GILL, D. O.; BAKER, D. M.; DUDA, M. G.; HUANG, X-Y; WANG, W.; POWERS, J. G. A Description of the Advanced Research WRF Version 3, NCAR Technical Note, 2008,125 p.

WATSON, A.I.; HOLLE, R.L.; LOPEZ, R.E. Cloud-toGround Lightning and Upper-Air Patterns during Bursts and Breaks in the Southwest Monsoon. Monthly Weather Review, v.122, n.8, p. 1726-1739, 1994.

ZEPKA, G. S. Previsão de descargas atmosféricas usando o modelo de mesoescala WRF. 2011. 176f. Tese (Doutorado em Geofísica Espacial) - Instituto Nacional de Pesquisas Espaciais, São José dos Campos.

ZHOU, J.; LAU, K-M. Does a Monsoon Climate Exist over South America? Journal of Climate, v. 11, n.5, p. 1020-1040, 1998. 